\title{
Constant errors occur in matched reproduction of angles even when likely biases are eliminated
}

\author{
A. W. MacRAE and H. K. LOH \\ University of Birmingham, Birmingham B15 2TT, England
}

\begin{abstract}
The literature on the reproduction of visually perceived angles often seems to contain an explicit or implicit assumption that systematic errors are caused by misperception of the sizes of the angles. We argue that in the absence of procedural artifacts no consistent error should arise from purely perceptual causes. Accordingly, an experimental procedure was designed to eliminate or evaluate a number of artifacts that probably affected earlier experiments. A computer-controlled display generated both a standard angle and a variable one that could be adjusted by a subject to match the standard. The data analysis followed Cox's (1970) procedure for the analysis of binary data to focus on the numbers of positive and negative errors rather than on their magnitudes and so eliminate the numerically distorting effects of asymmetrical error distributions. Nevertheless, the results showed what has usually been reported before: acute angles were matched with too large a comparison angle, and obtuse ones with too small an angle. Thus, there is a real effect to be explained, although the explanation must invoke response processes.
\end{abstract}

Attempting to test an allegedly widely held belief that acute angles are perceptually underestimated and obtuse ones are overestimated, Jastrow (1892) had subjects view an angle for 5 to $15 \mathrm{sec}$ and then immediately reproduce it from memory by adding a line to a horizontal line on a separate sheet of paper. Three of his subjects also reproduced the angle while the original was still in view, and these results differed only in that errors were smaller with simultaneous viewing. The patterns of error were otherwise equivalent.

The logical flaw in this procedure has been pointed out by Fisher (1969) (italics in original): "Suppose, for example, that when shown a $40^{\circ}$ angle a subject judges it to be one of $45^{\circ}$. When attempting to reproduce it he will construct two lines which he believes to represent an angle of $45^{\circ}$. What, therefore, will be the size of the ensuing angle? The outcome of Experiment 3 indicated that a given angle is constructed smaller in size than the one required. But in Jastrow's study the size of the angle was judged rather than specified. Without having first having evaluated the distortions arising in these two quite different situations, the only prediction open to Jastrow was that no distortion should have become apparent in the experiment which he performed" (p. 364).

In one experiment, Fisher obtained numerical estimates of the sizes of angles, and in another, he had subjects draw angles of numerically specified sizes. By combining both sets of results, he generated a resultant error pattern that closely resembled Jastrow's.

But Fisher's procedure is open to serious objection too. For one thing, if we take seriously the as- sumption he seems to share with Jastrow that the origin of the error is perceptual, then the conclusion to be drawn is that simultaneous matched reproduction should result in no net error. He proposes no theoretical reason why the errors in judgment and reproduction should not be exactly complementary. A different phrasing of Fisher's point in Robinson's (1972) critique of Jastrow's experiment emphasizes that conclusion: "After the subject had viewed each card it was covered and he had to reproduce the angle. Now by this procedure, subjects, however they over- or underestimated the angle perceptually, should have made accurate reproductions because, to draw an angle that looked like the one they saw, they would have to draw one the same actual size. If they overestimated the angle and began to draw it bigger, it would look even bigger because of their overestimation and so clearly be too big. This method will not get at the problem. Jastrow ought not to have found either over- or underestimation. That he did is probably evidence that some other factor, for example orientation of the lines, enters into the illusion" (p. 82).

Robinson goes on to add a criticism of Fisher's rationale: "The method he used was one of absolute judgment. Subjects were shown an angle and were asked to give it size in degrees. Fisher considered that this method was not prey to Jastrow's difficulties. I do not agree. Subjects learn the labels they attach to angles during a life-time's dealing with the angles they see. Thus, the label 'forty-five degrees' will be given to their own image, however distorted, of an angle of $45^{\circ}$ " (p. 82).

But if we fully accept Robinson's assertion, we 
should conclude not only that there should be no error in matched reproduction but that there should be none in absolute judgment or in production either, since the mental labeling of the perceived sizes of angles would compensate for the misperception.

If Fisher's proposal is dubious in the case of delayed reproduction, it is much more so in the case of simultaneous matched reproduction. With both angles visible, it is inconceivable that subjects would undertake a numerical estimate and then attempt to produce their estimated angle without reference to the original. Discrimination of differences is so much more precise than identification of absolute sizes that it is likely that subjects will focus on differences wherever possible. The matching process then becomes analogous to a discrepancy-reducing servo system. Of course, such a strategy can operate only insofar as the difference between the angles can readily be extracted from the display. Conditions for doing so are optimal when the two angles are superimposed, and they become worse when the provided and the reproduced angles are separated in position, direction, or time.

Of course, the logical difficulties raised above relate only to the interpretation of the results. The actual results stand and require explanation. If perceptual overestimation or underestimation cannot account for Jastrow's results and for the concordant findings by Beery (1968) and Maclean and Stacey (1971), how are they to be explained?

One possible cause of perceptual difference between the provided and the reproduced angle is that in every case cited they were indeed objectively different in appearance. Jastrow's subjects viewed angles drawn on circular cardboard and drew them on squares of paper. Until the subject drew a second line, only an imaginary or a partial response angle could be compared with the provided one-and after it was drawn, no adjustment was possible. Beery's arrangement was similar, except that the stimulus angle was drawn in purple ink and the same sheet of paper was used for the subject's freehand, penciled response. Maclean and Stacey projected their stimulus angles on a screen, and subjects had to draw the second arm of the angle on a sheet of paper on which one arm was provided.

The location of the stimulus angle was not specified by Jastrow. Beery had the stimulus and response angles coplanar and vertically adjacent, but Maclean and Stacey had the stimulus angle in a vertical plane and the response angle in a horizontal plane. Such an arrangement places heavy reliance on shape constancy, rather unfortunately since perceptual error is the subject under investigation.

The experiment reported here avoided these problems. The stimulus and response angles were made coplanar and equal in color and brightness by generating both on the face of a computer-controlled display screen.

Unless the fixed and the adjustable angle are adjacent, you cannot be confident that the situation is truly one of simultaneous matched reproduction. In the arrangement used by Maclean and Stacey, it seems quite unlikely that subjects could in fact view both angles simultaneously, since they were at very different distances, in different planes, and in different directions from the subjects' eyes. Since the method of angle reproduction by drawing makes no provision for correction or adjustment of the completed response, it is possible that the inevitable time interval between last viewing the stimulus and completing the response plays some part in bringing about errors. In the experiment reported here, the subject could continue adjusting the response angle until satisfied with the result. Therefore, in the simultaneous reproduction condition, no time delay imposed itself. To evaluate the effects of delay, other experimental conditions provided $3 \mathrm{sec}$ exposure of the stimulus angle, followed by the appearance of the response angle after delays of 0,1 , or $4 \mathrm{sec}$.

Orientation of the angles was found by Fisher to affect absolute judgments, while Beery and Maclean and Stacey found that it affected matched reproduction. However, their procedure entailed providing one arm of the response angle, so their subjects may have approached the task with the objective of achieving a correct alignment of the second arm rather than of generating an angle of the correct size. Although both objectives would lead to the same outcome if successful, they may not be subject to the same errors. Furthermore, any error would produce a change in alignment of the angle's imaginary bisector as well as an angle discrepancy.

In addition, in the matched reproduction task it was not possible to distinguish between the effect of varying the orientation of the stimulus angle and that of the response angle, since they varied together. All of these problems were avoided in the present experiment.

\section{METHOD}

\section{Apparatus}

A PDP-11/20 computer generated two angles on a Tektronix 611 storage oscilloscope operating in refresh mode. The lines forming the angles were $29 \mathrm{~mm}$ long and were made up of discrete dots at approximately $2-\mathrm{mm}$ intervals to avoid the flicker that became troublesome with larger numbers of points. The vertex of one angle was $45 \mathrm{~mm}$ above and $45 \mathrm{~mm}$ to the left of the other, the vertices being symmetrically placed about the center of the $165 \times 212 \mathrm{~mm}$ screen.

A subject viewed the screen from a freely chosen, comfortable viewing distance and controlled one of the angles by means of a rotary potentiometer in a small control box held in one hand 
and manipulated with the other. Rotating the potentiometer knob caused both arms of the angle to rotate symmetrically about the vertex, so that the angle's imaginary bisector remained constant in direction. The knob's maximum rotation of $150 \mathrm{deg}$ corresponded to a 170-deg change in the size of the displayed angle. On a random half of all trials, a clockwise rotation of the knob increased the size of the angle from a lower limit of $10 \mathrm{deg}$ to an upper limit of $180 \mathrm{deg}$. On the remainder, the displaycontrol relation was reversed.

\section{Procedure}

To initiate a trial, the subject turned the control knob fully anticlockwise. The standard angle appeared as soon as this had been done. The response angle appeared either simultaneously or following the disappearance of the standard. When the response angle appeared, it was either at $10 \mathrm{deg}$, which could be increased to $180 \mathrm{deg}$ by rotating the knob fully clockwise, or at $170 \mathrm{deg}$, which could be reduced to $0 \mathrm{deg}$ by the same clockwise rotation of the knob. In practice, the subjects rarely or never adjusted the response angle to the most extreme settings that were available. These conditions occurred equally often but in random order.

The design strategy of using two starting points for adjustment was dictated by the following considerations. The starting point may well have some influence on the final setting of the adjustable angle. For example, successive contrast may increase the apparent size (and thus decrease the preferred setting) of the variable angle when adjustment begins from a smaller angle, and decrease its apparent size when adjustment begins with a larger one. Alternatively, perseverance effects may lead to just the opposite kind of error.

If adjustment always begins from, say, $90 \mathrm{deg}$, then any such effect will operate in one direction with acute angles and in the opposite direction with obtuse angles. That could wholly account for any relationship between error and angle size that emerged, and there would be no possibility of evaluating its importance. By adopting two starting points lying outside the range of angles to be matched, the influence of starting point is not eliminated, but at least it can be analyzed.

Of course, one might learn more by using more than two starting points, but the experiment followed a full factorial design, so even three starting points would have required the two sessions to last about 90 min each instead of the $1 \mathrm{~h}$ that we judged to be all we could ask of our subjects.

The fixed angle was randomly selected on each trial to be one of eight sizes from 20 to $160 \mathrm{deg}$ in 20 -deg steps. The orientations of the imaginary angle bisectors were randomly and independently selected to be horizontal with the vertex on the left or vertical with the vertex below. In the simultaneous matching condition, both angles appeared at the same time and both remained in view until the subject indicated, by pressing a button on the control box, that a satisfactory match had been achieved. The size of the response angle was then recorded on disk, and the display was blanked. The subject was free to decide when the next trial should begin. In three other timing conditions, the stimulus angle appeared alone for $3 \mathrm{sec}$ and then disappeared. The response angle appeared after a delay of 0,1 , or $4 \mathrm{sec}$, and the subject completed the trial as in the simultaneous condition. These four timing conditions all occurred equally often and in random order in each session.

Each experimental session thus had 256 combinations of conditions, made up of 2 stimulus angle orientations $\times 2$ response angle orientations $\times 2$ starting points $\times 4$ timing conditions $\times 8$ angles. Conditions were selected by a subroutine that selected randomly without replacement from the set of all those available. Thus, each of these combinations occurred once in an experimental session in a random order that was different in every case.

In any one session, the adjustable response angle was either the upper or the lower one throughout, but all subjects per- formed one session in each of these conditions, half in one order and half in the other. Each session lasted about $1 \mathrm{~h}$.

\section{Subjects}

Six male and six female undergraduates volunteered as subjects without payment.

\section{RESULTS}

It will be argued that experiments such as this are usually analyzed in a way that is inadequate and misleading, but for comparability with other studies a traditional summary of the results is shown in Figure 1.

There is an overall tendency for positive errorsthat is, excessively large settings of the response angle-to occur with acute angles and for negative errors to occur with obtuse angles. However, negative errors with angles of 40 and 60 deg occurred in the delayed conditions, especially when the standard angle had its midline vertical or the response angle had its midline horizontal. This effect appears at first sight to be an instance of the horizontal-vertical illusion, in which vertical distances appear greater than physically equivalent horizontal ones. Such an effect would operate to make a horizontally oriented angle look larger, thus giving rise to a positive error if that angle was the standard and a negative error if the response angle was the one being perceptually overestimated, since an angle that looked larger than it should would have to be adjusted to a size that was too small in order for its appearance to be correct. Now the horizontal-vertical illusion should apply equally to acute and to obtuse angles, but that is not what we find here. In every condition, the HV display and the VH display, which should show the greatest difference under the influence of any such effect, reverse their relative error directions when the angle changes from acute to obtuse. The horizontal-vertical illusion would correctly predict the results we observe with the acute angles but makes the wrong prediction for the obtuse ones. Thus, the horizontal-vertical illusion does not give an adequate account of these results.

But, rather than undertake further interpretation of the mean errors, we would prefer to explain why we consider them to be misleading and introduce an alternative analysis.

Examination of the error distributions shows them to be markedly asymmetric for the largest and the smallest angles. The asymmetry may have more than one cause, but the simplest explanation is that there is just more room to err in one direction than the other. A related effect is that a 15-deg angle differs more noticeably from $20 \mathrm{deg}$ than does an angle of $25 \mathrm{deg}$. Thus, even when there is no systematic tendency to err in one direction rather than another, it should be expected that the random errors in one 

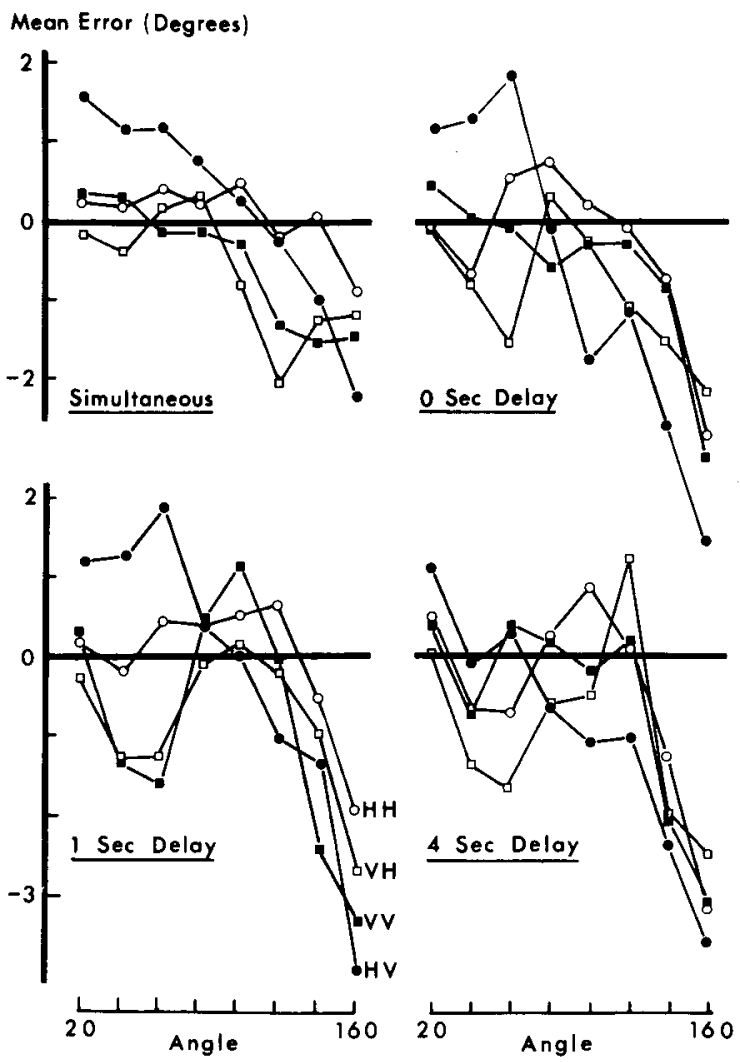

Figure 1. Mean error in the size of a variable angle when attempting to match the size of a standard angle. Standard angles ranged in 20-deg steps from 20 to $160 \mathrm{deg}$. In Condition HH both angles had horizontal midlines, and in Condition VV they were both vertical. In HV the standard angle was horizontal and the variable was vertical, while in VH the reverse was true. Each plotted point is the mean error by 12 subjects in four conditions, made ap of each combination of two starting point relations and two arrangements of the angles on the display.

direction, even if no more numerous, should be larger than those in the other direction. Such a disparity in error magnitudes would produce positive errors in the means of the responses to the smallest angles and negative errors in the means of the largest. Examination of the distributions of error showed that such a tendency did exist. Small angles gave errors skewed positively, whereas the largest angles had negatively skewed error distributions.

Interpretation of the means is thus likely to be misleading, and it is necessary to seek an analysis that will be insensitive to skew in the error distributions. A first step is merely to count errors as positive or negative without regard to size. Totaling the positive errors over the 12 subjects yields a single number for every one of the 512 combinations of experimental treatments. If no effects other than the skew in error sizes operate, these totals will differ only randomly from 6 (half of the subjects making positive errors and half making negative ones).
For further analysis, we follow Cox (1970) and apply an empirical logistic transformation, $Z=\log$ $[(P+.5) /(N+.5)]$, where $P$ is the number of positive errors and $\mathbf{N}$ is the number of negative errors. The resultant $\mathrm{Z}$ scores are analogous to scaled and normalized mean errors. Figure 2 shows the same features as Figure 1, but expressed in $\mathrm{Z}$ scores.

For easier interpretation, the Z-score ordinates have been labeled using the proportions that the $\mathrm{Z}$ scores represent. The graphs thus display proportions of positive errors that have been averaged logistically across four combinations of spatial arrangement and starting point.

The most striking, and perhaps the most important, difference between Figure 1 and Figure 2 is the global difference between the two measures of error as a function of time delay. Figure 1 shows the mean errors to be smallest in the simultaneous viewing condition, whereas the logistically transformed proportions in Figure 2 show their largest effects with simultaneous viewing, and a progressive decline in deviations from zero as response delay increases.

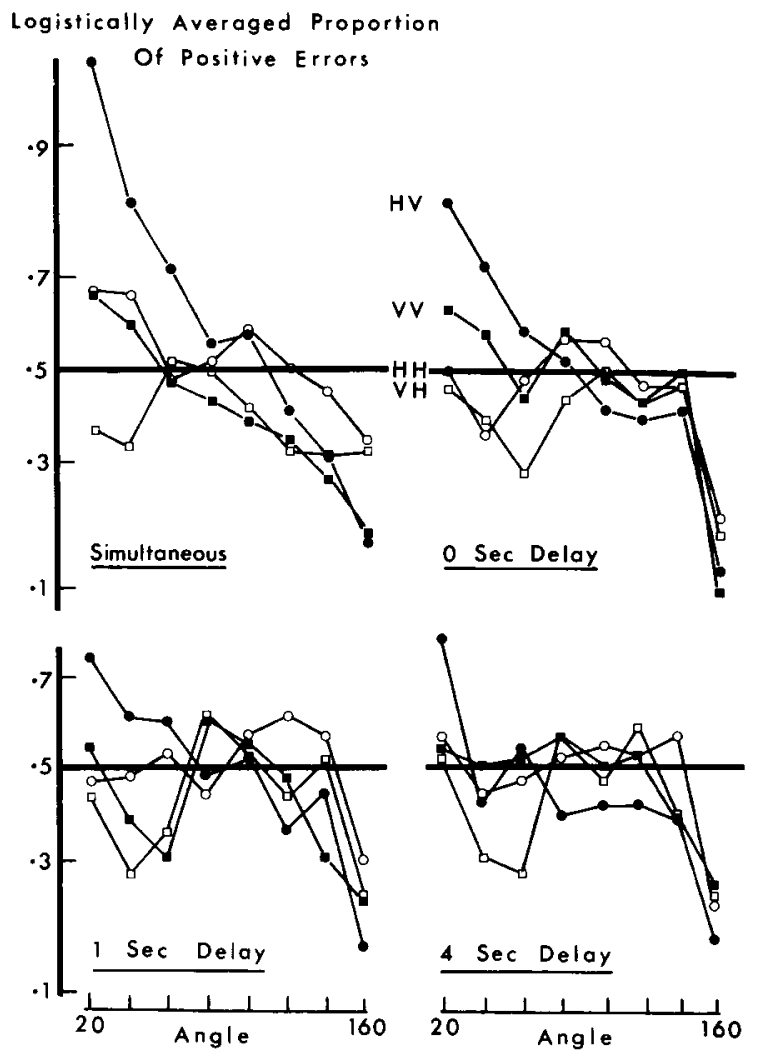

Figure 2. The same data as in Figure 1 are here treated differently. Each plotted point is the mean over the four combinations of starting point and spatial arrangement of the logistically transformed proportion of positive errors. For greater ease of interpretation, the vertical axis has been labeled in units of proportion rather than in logistic deviates. 
The difference substantiates our suspicion that mean error is a misleading measure for this task. As delay increases, the task becomes more difficult and the responses become more variable. And it is the increase in random variability that causes the opposing trends in Figures 1 and 2 . In the simultaneous condition, errors are relatively small but consistent, with the result that overestimation is shown by very large or very small numbers of subjects, depending on the direction of the constant tendency. Thus extreme $\mathrm{Z}$ values are generated. As variability increases and the random component in the errors become more important, the proportions of overestimates and underestimates tend towards .5 since the systematic component has less influence. This has the effect of reducing the absolute size of the logistic scores. At the same time, the variance and range of error increases and the asymmetrical shape of the error distribution has a greater biasing effect on the means, which therefore show more extreme values.

Note that, when the error distribution is skewed, random variation alone will always give rise to apparently systematic mean errors but it cannot give rise to systematically unequal error proportions, so it is clear that in a case of conflict between the measures the latter is to be preferred. No other effect observed in Figure 1 is seriously contradicted by Figure 2 . In particular, the tendency to positive errors with acute angles and to negative errors with obtuse angles can be seen in both, as can the crossover of the lines for VH and HV. The latter effect is one for which we can offer no explanation. It can be described differently by saying that angles with horizontal midlines tend to look more like right angles than they should, while those with vertical midlines look less like right angles than they should, but such a rephrasing is of course no explanation.

One must be cautious in interpreting the results of Maclean and Stacey, since some of their data summaries represent dubious averages across absolute judgment and matched reproduction conditions. However, their Figure 4, showing mean error as a function of angle in conditions corresponding to our $\mathrm{HH}$ and VV, is in good agreement with our simultaneous condition curves in Figures 1 and 2. They all show a moderate tendency to positive errors for very acute angles in both orientations and a tendency to negative errors for obtuse angles, especially when they are vertical.

By our method we sought to eliminate procedural artifacts we suspected in earlier studies, and by our analysis we avoided a computational one. We used four time conditions to trace any effect on matching performance of nonsimultaneous viewing of the standard and the variable, and we used two starting points to evaluate effects relating to the direction in which adjustments were made. But our results force us to conclude that errors in simultaneous matched reproduction really do occur when these artifacts are eliminated. And imposing a delay on reproduction serves only to obscure the effect. Consequently, our further analyses relate only to the condition with simultaneous, unlimited viewing of the standard and variable.

The logistic analysis of the data provides a simple means of evaluating the significance of effects. The variance of each logistic deviate can be calculated a priori from the formula $V=(n+1)(n+2)$ / $[n(P+1)(N+1)]$, where $n=P+N$ and $V$ is the variance of the estimate. Contrasts, each with $1 \mathrm{df}$, are calculated by summing the products of each logistically transformed proportion and its appropriate contrast coefficient. The variance of a contrast is found by summing the products of the corresponding variances and the squares of the contrast coefficients. Thus, the contrast divided by the square root of its variance provides a standardized contrast, $z$, which can be compared with normal curve tables. For the rationale and some worked examples, see Cox (1970). Strictly, this style of analysis applies when the various ns represent groups of different individuals, but the effect of having the same individuals in each group is just to make our significance tests somewhat more conservative.

The effect of angle was represented in the analysis by its linear, quadratic, and cubic trend components. Up to seven components could of course be examined, but neither the quadratic nor the cubic component, nor any of their interactions with other factors, approached the $5 \%$ level of significance. On the other hand, the linear component was enormously significant $(z=9.56, p \ll .001)$, reflecting the decrease in the average proportion of positive errors as the angles increased in size. The linear component also interacted significantly with the orientations of the standard and the variable $(z=3.33$ and 5.91 , respectively, $\mathrm{p}<.001$ ). These effects corresponded to the puzzlingly different average slopes of the four lines in the "simultaneous" section of Figure 2. Two factors in the experiment are not represented in Figure 2 because it averages data across the two values used as starting points for adjustment and across the two spatial arrangements. The starting point had little impact in the simultaneous condition $(z<1.00$ for the main effect and all its interactions with other factors, including all the trends relating to angle), although it exerted much more influence in the delayed reproduction conditions in which, in the absence of the standard angle, subjects could perhaps be more easily affected by the starting value set for the response angle. Nor was there any indication that small or large starting values had differential effects on the settings of large and small angles, because any such effect would 
have revealed itself as an interaction between starting point and linear trend in angle.

Thus, it appears that in the simultaneous matching condition the biasing effects of successive contrast and of perseverance did not appreciably affect the errors made. Either the effects were small or opposing effects canceled one another.

The spatial arrangement of the angles on the screen had negligible effect on average $(z=.16)$, though it did interact significantly with the orientation of the variable angle $(\mathrm{z}=3.58, \mathrm{p}<.001)$. In addition, the only significant three-way interaction involved spatial position, orientation of the variable, and the linear trend component in angle size $(z=3.38$, $\mathrm{p}<.001)$.

\section{DISCUSSION}

The experiment was undertaken to eliminate or evaluate artifacts we believed to be responsible for reported systematic errors in matched reproduction of angles, but we found that constant errors occurred even with our procedure.

One of our objectives had been to eliminate the great distance between standard and variable that occurred in some other studies. To superimpose the angles would trivialize the task, so we opted for having the angles close together but not horizontally or vertically aligned. Now it is clear from the results that the relative locations of standard and variable had an important bearing on the errors that were made. One probable reason is that in some combinations of conditions an arm of one angle might be quite close to an arm of the other. In some configurations, too, an imaginary continuation of the arms of one angle would partially enclose the other angle. Such effects might alter the appearance of both angles. But they could not account for most of the results, because the standard and the variable were equally often in the upper left position, so that any tendency to overestimate the upper one, say, was counterbalanced across the other conditions. It is for this reason that position has a negligible effect on average but shows some strong interactions.

But the principal result to be explained is the linear trend in error as a function of angle-the highly significant tendency for acute angles to be set too large and for obtuse ones to be set too small. No purely perceptual error can account for this result for the reasons set out earlier. Any explanation must invoke response processes, for example, a greater tolerance for error in one direction once the error has occurred. Such unequal sensitivity to error could relate to our different acuity for inclinations close to and remote from the major axes, or it might be related to Weber's law. However, the present experiment was conceived with other objectives in mind and is not well suited to provide evidence on these speculations.

The present experiment and the others we have cited looked at subjects' performances on anglematching tasks in a global, functional way. Subjects have been permitted a free choice of viewing distance and even of head orientation to allow them to choose the conditions of viewing that seemed to them to offer most comfort and most accuracy. They also had a free choice of response strategy and of response timing. By contrast, another line of work with antecedents in retinal physiology sets out to control closely the stimulus configuration actually stimulating a subject's retina by requiring a fixed head position.

Of course, some experimental questions about the causes of error require such constraints on the subjects, but there seems to us to be a danger that the constraints may sometimes cause a subject to make errors he might have avoided. We think it less surprising to find consistent patterns of error in these constrained tasks than in our task, in which subjects were free to adopt their own preferred modes of viewing and responding.

\section{REFERENCES}

BeEry, K. E. Estimation of angles. Perceptual and Motor Skills, 1968, 26, 11-14.

Cox, D. R. The analysis of binary data. London: Methuen, 1970.

Fisher, G. H. An experimental study of angular subtension. Quarterly Journal of Experimental Psychology, 1969, 21, 356366.

JASTrow, J. On the judgment of angles and positions of lines. A. On the judgment of angles. American Journal of Psychology, $1892,5,214-217$.

Maclean, I. E., \& Stacey, B. G. Judgment of angle size: An experimental appraisal. Perception \& Psychophysics, 1971, 9, 499-504.

RoBinson, J. O. The psychology of visual illusion. London: Hutchinson, 1972.

(Manuscript received August 28, 1980; revision accepted for publication June 30,1981 .) 